\title{
Prevalência de pressão arterial elevada em escolares e adolescentes de Maceió
}

\author{
Prevalence of high blood pressure in children and adolescents from the city of Maceió, Brazil
}

\author{
Adriana A. Moura1, Maria A.M. Silva2, Maria R.M.T. Ferraz ${ }^{3}$, Ivan R. Rivera4
}

\section{Resumo}

Objetivos: Definir a prevalência de pressão arterial elevada em amostra representativa de escolares e adolescentes de Maceió (Alagoas, Brasil) e pesquisar a associação de pressão arterial elevada com idade, gênero e estado nutricional.

Métodos: Estudo epidemiológico descritivo, transversal, randomizado, realizado entre maio de 2000 e setembro de 2002, que avaliou indivíduos de 7 a 17 anos sorteados entre 185.702 alunos de escolas públicas e privadas de Maceió. O cálculo da amostra foi realizado com base no valor esperado da prevalência de hipertensão arterial sistêmica para a faixa etária. Após a randomização, os dados foram coletados através de questionário, aferição de peso e altura e duas medidas da pressão arterial. Pressão arterial elevada foi definida como pressão arterial sistólica e/ou diastólica igual ou acima do percentil 95 em qualquer das duas medidas realizadas.

Resultados: A amostra final constituiu-se de 1.253 estudantes (706 do gênero feminino). Foram identificados 118 estudantes com pressão arterial elevada, média de idade de 13 anos, sendo $44 \%$ do gênero masculino. Risco de sobrepeso foi identificado em $9,3 \%$ das crianças, e sobrepeso, em 4,5\%; houve associação significante dessas variáveis com pressão arterial elevada.

Conclusões: A prevalência de pressão arterial elevada foi de $9,4 \%$, e foi significantemente maior nos estudantes com sobrepeso e com risco de sobrepeso.

J Pediatr (Rio J). 2004;80(1):35-40: Pressão arterial, prevalência, escolares, adolescentes.

\section{Introdução}

A hipertensão arterial sistêmica (HAS) é uma doença definida pela persistência de níveis de pressão arterial acima de valores arbitrariamente definidos como limites de normalidade ${ }^{1}$. É a doença cardiovascular mais comum, considerada o maior desafio de saúde pública para sociedades em transição socioeconômica e epidemiológica e um dos mais importantes fatores de risco de mortalidade cardiovas-

1. Mestre em Saúde da Criança, Universidade Federal de Alagoas (UFAL).

2. Professora adjunta, Departamento de Clínica Médica, UFAL.

3. Mestre em Saúde da Criança, UFAL.

4. Professor adjunto, Departamento de Tocoginecologia e Pediatria, UFAL.

Fonte financiadora de equipamento e materiais: Fundação de Amparo à Pesquisa do Estado de Alagoas (FAPEAL).

Artigo submetido em 16.04.03, aceito em 03.11.03.

\section{Abstract}

Objectives: To define the prevalence of high blood pressure in a representative sample of children and adolescents from the city of Maceió, state of Alagoas, Brazil, and to investigate the association of high blood pressure with age, sex and nutritional status.

Methods: This cross-sectional study was carried out from May 2000 to September 2002. Individuals between 7 and 17 years of age were selected among all the 185,702 students from public and private schools. The size of the sample was defined based on the expected prevalence of hypertension for the age group. After randomization, data were collected through a questionnaire. Blood pressure was measured twice. Weight and height were also measured. High blood pressure was defined as systolic and/or diastolic blood pressure over the $95^{\text {th }}$ percentile in one or in both measures.

Results: The final sample included 1,253 students ( 706 females). One hundred and eighteen students had high blood pressure (mean age 13 years; $44 \%$ males). Risk of being overweight and excess weight were identified, respectively, in 9.3 and $4.5 \%$ of the students. These variables were significantly associated with high blood pressure.

Conclusions: The prevalence of high blood pressure was $9.4 \%$. High blood pressure was significantly more frequent among overweight students and among those at risk for being overweight.

J Pediatr (Rio J). 2004;80(1):35-40: Blood pressure, prevalence, children, adolescents.

cular, sendo responsável por $20-50 \%$ de todas as mortes ${ }^{2}$. No Brasil, a HAS afeta 14 a $18 \%$ da população adulta ${ }^{3}$.

Em lactentes e pré-escolares, a hipertensão é incomum e, quando presente, geralmente indica um processo patológico subjacente. Escolares e, em particular, adolescentes, podem apresentar hipertensão primária ou essencial, que usualmente é detectada através de avaliação rotineira da pressão arterial. Esta é atualmente a principal causa de hipertensão arterial nessa faixa etária4-8.

Muitos estudos têm revelado fortes indícios de que a HAS do adulto é uma doença que se inicia na infância, ${ }^{4-6,9,10}$ o que tem aumentado a preocupação com a avaliação da pressão arterial em crianças nas últimas décadas.

Em 1977, nos Estados Unidos, foi publicado o primeiro relatório da força-tarefa sobre o controle da pressão 
arterial em crianças ${ }^{4}$, que apresentava uma proposta de padronização do método de medida e das curvas de distribuição da pressão arterial em crianças normais, organizadas em gráficos de percentis de acordo com a idade e com o sexo. Neste estudo, o percentil 95 ficou estabelecido como o limite de normotensão, e a recomendação de que "todo pediatra deverá medir a pressão arterial das crianças maiores de 3 anos no mínimo uma vez ao ano e acompanhá-la anualmente em um gráfico de percentis" permanece válida até hoje.

Desde então, uma série de estudos nacionais e internacionais vêm sendo desenvolvidos, e novos conceitos vêm sendo elaborados. Em 1987, foi divulgado o segundo relatório da força-tarefa sobre o controle da pressão arterial em crianças $^{5}$. Em 1993, Rosner et al. publicaram uma metaanálise com tabelas de referência para pressão arterial normal (abaixo do percentil 90), normal-alta (entre o percentil 90 e o percentil 95) e alta (acima do percentil 95), por idade e por sexo, subdivididas em percentis de altura, a partir de 76.018 medidas de pressão arterial ${ }^{10}$. Além disso, passaram a utilizar e também a recomendar a fase $V$ de Korotkoff (K5) como referência para pressão diastólica para todas as idades. Em 1996, foi publicada a atualização do segundo relatório da força-tarefa de $1987^{9}$.

No Brasil, Alves et al., em 1988, investigaram a freqüência de hipertensão arterial em um grupo de 989 préescolares e escolares no Recife, todos de boa condição socioeconômica, e detectaram um percentual de $2,12 \%$ de crianças hipertensas, utilizando as normas do grupo da força-tarefa de $1987^{11}$. Em 1995, Oliveira avaliou 1.005 alunos randomizados entre os estudantes regularmente matriculados em escolas públicas e privadas de Belo Horizonte, realizando exame antropométrico e duas medidas de pressão arterial. O autor encontrou valores da pressão arterial sistólica e diastólica discretamente inferiores aos da referência da força-tarefa de $1987^{12}$.

Este trabalho tem como objetivos: 1) definir a prevalência de pressão arterial elevada em escolares e adolescentes da cidade de Maceió; 2) pesquisar a associação de pressão arterial elevada com variáveis como idade, gênero e estado nutricional.

\section{Casuística e métodos}

O presente trabalho é um estudo epidemiológico, descritivo, observacional, em corte transversal, realizado no período de maio de 2000 a setembro de 2002. A amostra consistiu de escolares e adolescentes que freqüentavam as escolas de nível fundamental e médio de Maceió, Alagoas, de ambos os sexos, com idades de 7 a 17 anos.

Para definir a prevalência de pressão arterial elevada em crianças e adolescentes na cidade de Maceió, de forma que os valores encontrados representassem a população em estudo, o cálculo da amostra foi realizado com base num valor esperado para a prevalência de HAS, na faixa etária considerada, utilizando o III Consenso de Hipertensão Arterial da Sociedade Brasileira de Cardiologia, de
1998: $7 \%{ }^{13}$. Assumiu-se um intervalo de confiança de $95 \%$ e uma precisão de $2 \%$. O tamanho final da amostra foi de 1.253 indivíduos.

A randomização foi realizada mediante uma amostragem por conglomerados, sendo cada escola considerada um conglomerado. Escolheu-se trabalhar com 40 escolas para que se obtivesse melhor precisão nas estimativas de prevalência encontradas e uma melhor distribuição demográfica dos alunos pesquisados.

Após o sorteio, a distribuição das escolas foi a seguinte: uma federal, 20 estaduais, oito municipais, 11 particulares.

Como as escolas sorteadas tinham tamanhos diferentes, para obter uma representação na amostra final que fosse proporcional ao tamanho de cada uma, foram sorteados $2,7 \%$ dos alunos nelas matriculados; estes, somados, completariam os 1.253 indivíduos necessários. O consentimento para a participação no estudo foi obtido das escolas sorteadas e dos pais dos indivíduos selecionados.

Os dados foram coletados sob a forma de questionário e medidas da pressão arterial, do peso e da altura do indivíduo. A medida da pressão arterial foi realizada apenas por uma pesquisadora médica, a qual recebeu treinamento específico.

Os estudantes que se recusaram a participar da pesquisa foram substituídos utilizando-se o mesmo processo de randomização, de forma a manter o tamanho previsto da amostra.

Com relação aos parâmetros utilizados, a pressão arterial foi considerada elevada quando a medida da pressão arterial sistólica e/ou diastólica era igual ou superior à encontrada no percentil 95 nas tabelas de referência do Update on the 1987 Task Force Report on High Blood Pressure in Children and Adolescents, de $1996^{\circ}$, para idade e sexo correspondentes, ajustados para o percentil de estatura do avaliado. Para a definição do percentil de estatura do indivíduo, foram utilizados os gráficos de estatura por idade e sexo do Centro Nacional de Estatísticas de Saúde Norte-Americano ${ }^{14}$. Foram realizadas duas medidas de pressão arterial, com intervalo mínimo de dois minutos. Pressão arterial igual ou acima do percentil 95 em qualquer uma das duas medidas serviu para definir a prevalência de pressão arterial elevada. Foi utilizado um esfigmanômetro de coluna de mercúrio, avaliado quanto à fidedignidade no Instituto de Metrologia Brasileiro (INMETRO), com manguitos de duas dimensões diferentes ( $25 \times 12 \mathrm{~cm} \mathrm{e} 18 \times 9 \mathrm{~cm}$ ) e um estetoscópio pediátrico. Para as medidas de pressão arterial, foram seguidas as recomendações metodológicas do III Consenso Brasileiro de HAS de $1998^{13}$ e do Task Force de 1996 (Tabela 1). Para a avaliação do estado nutricional, foi utilizada a classificação do CDC (Centers for Disease and Control and Prevention), Atlanta, 2001, que é baseada no índice de massa corpórea (IMC): baixo peso, IMC inferior ao percentil 5; peso normal, IMC superior ao percentil 5 e inferior ao percentil 85; risco de sobrepeso, IMC igual ou superior ao percentil 85; sobrepeso, IMC igual ou superior ao percentil $95^{15}$. Para a medida do peso, utilizou-se uma balança digital para até $150 \mathrm{~kg}$ calibrada e aferida pelo 
INMETRO; para a estatura, foi utilizado um estadiômetro com um cursor, ambos de madeira, graduado com precisão de $0,1 \mathrm{~cm}$.

Os dados obtidos durante a coleta foram armazenados num programa de banco de dados (Microsoft ${ }^{\circledR}$ Excel 2000) e analisados utilizando o programa Epi-Info 200017. Foi utilizada análise de variância simples para comparar diferenças de médias de pressão arterial sistólica ou diastólica entre grupos por gênero, faixa etária e estado nutricional. Foram utilizados os testes de qui-quadrado $\left(\chi^{2}\right)$ e de Fisher para comparar diferenças de prevalência de pressão arterial normal-alta ou alta nos mesmos grupos. Foi estabelecido como nível de significância estatística para rejeição de uma hipótese nula um $\mathrm{p}<0,05$ ou $5 \%$ para todos os testes.

O protocolo de pesquisa foi aprovado previamente pelo Comitê de Ética do Hospital Universitário da Universidade Federal de Alagoas.

\section{Resultados}

A amostra final foi constituída por 1.253 escolares e adolescentes, com idades de 7 a 17 anos e média de 12,4 $\pm 2,9$ anos, sendo 547 do gênero masculino e 706 do gênero feminino.

Pressão arterial elevada esteve presente em 9,41\% dos estudantes quando avaliadas as medidas isoladas (intervalo de confiança: 7,8-11,02). Quando considerada apenas a média das duas medidas, a prevalência foi de $7,7 \%$ (intervalo de confiança: 6,5-9,5).

A análise dos questionários demonstrou que os estudantes com pressão arterial elevada não se encontravam em uso de drogas que elevam a pressão arterial nem possuíam, no momento da investigação, diagnóstico de doenças que cursam com hipertensão arterial.
Em relação à prevalência de pressão arterial elevada, não foram observadas diferenças estatisticamente significantes entre os gêneros: 9,5\% (52) no sexo masculino e $9,3 \%(66)$ no sexo feminino $(p=0,95)$. Também não foi observada diferença estatisticamente significante quando comparados indivíduos de diferentes gêneros com a mesma faixa etária. Quando não subdivididos em relação ao gênero, os estudantes apresentaram diferença significante na comparação do grupo de 11-14 anos com o de 15-17 anos, e de 7-10 anos com o de 15-17 anos (Tabela 2).

Em relação ao estado nutricional, encontrou-se a seguinte distribuição na população estudada: peso normal, $86,3 \%$ (1.081); risco de sobrepeso, 9,3\% (116); e sobrepeso, $4,5 \%$ (56).

Em relação à prevalência de pressão arterial elevada, houve diferença extremamente significante quando comparados os grupos peso normal versus sobrepeso, significante quando comparados os grupos sobrepeso versus risco de sobrepeso, e não-significante quando comparados os grupos peso normal versus risco de sobrepeso (Tabela 3 ).

\section{Discussão}

Como doença do adulto de alta morbimortalidade que pode ter seu início na infância e na adolescência, a HAS tem sido uma das afecções mais estudadas em toda a medicina. Os estudos epidemiológicos têm grande importância nesse cenário, pois trazem informações sobre como essa entidade evolui com a idade e quais os fatores de risco envolvidos no surgimento e na manutenção de níveis tensionais elevados, bem como o modo como esses níveis desencadeiam estados mórbidos. Fatores genéticos, nutrição e estilo de vida vêm sendo cada vez mais implicados e correlacionados com níveis altos de pressão arterial ${ }^{18}$. Assim, pais, professores

Tabela 1 - Recomendações metodológicas do III Consenso Brasileiro de Hipertensão Arterial Sistêmica, de $1998^{13}$, e do Task Force, de $1996^{9}$, adotadas no presente trabalho

\begin{tabular}{ll}
\hline Item & Recomendação \\
\hline Largura do manguito & $\begin{array}{l}40 \% \text { da medida da circunferência do braço no ponto médio entre o cotovelo e o } \\
\text { acrômio }\end{array}$ \\
Comprimento do manguito & 80 a $100 \%$ da medida do braço \\
Manômetro & Coluna de mercúrio \\
Estetoscópio & Sobre pulso arterial braquial, $2 \mathrm{~cm}$ acima da fossa cubital \\
Paciente & Calmo, sentado, braço apoiado no nível do coração; medida após 3 a 5 minutos \\
de repouso & Duas, com 1 a 2 minutos de intervalo entre as medidas sucessivas \\
Número de medidas & Primeiro som de Korotkoff \\
Sístole & Quinto som de Korotkoff \\
Diástole &
\end{tabular}

Adaptado de $\mathrm{Koch}^{28}$. 
Tabela 2 - Prevalência de pressão arterial elevada em estudantes de diferentes faixas etárias de Maceió de ambos os gêneros

\begin{tabular}{lccc}
\hline Idade (N) & \multicolumn{2}{c}{ Prevalência por gênero } & \multirow{2}{*}{ Prevalência global } \\
& Masculino & Feminino & \\
\hline $7-10$ anos (355) & $4,2 \%(7)^{1}$ & $8,4 \%(16)$ & $6,5 \%(23)$ \\
$11-14$ anos (552) & $7,9 \%(18)^{2}$ & $9,5 \%(31)$ & $8,9 \%(49)$ \\
$15-17$ anos (346) & $17,6 \%(27)^{3}$ & $9,9 \%(19)$ & $13,3 \%(46)$ \\
\hline
\end{tabular}

\footnotetext{
1 Pressão arterial elevada masculino $x$ feminino, Fisher bi-caudal, $p=0,1324, \chi^{2}$ não-corrigido, $p=0,1679$

2 Pressão arterial elevada masculino $x$ feminino, Fisher bi-caudal, $p=0,5464, \chi^{2}$ não-corrigido, $p=0,6125$

3 Pressão arterial elevada masculino $x$ feminino, Fisher bi-caudal, $p=0,0396, \chi^{2}$ não-corrigido, $p=0,0541$

Pressão arterial elevada (7-10 x 11-14 anos), Fisher bi-caudal, $p=0,2104, \chi^{2}$ não-corrigido, $p=0,2449$

Pressão arterial elevada (11-14 x 15-17 anos), Fisher bi-caudal, $p=0,0336, \chi^{2}$ não-corrigido, $p=0,0376$

Pressão arterial elevada (7-10 x 15-17 anos), Fisher bi-caudal, $p=0,0023, \chi^{2}$ não-corrigido, $p=0,0034$
}

e pediatras devem investir nos aspectos relacionados à prevenção e ao seguimento da rotina de aferições anuais já propostas amplamente nos consensos de cardiologia e pediatria.

Em crianças, os níveis que definem pressão arterial elevada baseiam-se em limites estatísticos, ou seja, arbitrários $^{9}$. Por isso, medidas isoladas são úteis apenas para avaliações em momentos específicos. É necessário avaliar os níveis tensionais ao longo do crescimento da criança, descobrindo qual o percentil de pressão arterial que se correlaciona com esses valores ${ }^{9}$.

\section{Prevalência de pressão arterial elevada em escolares e adolescentes da cidade de Maceió}

Estudos nacionais e internacionais têm encontrado valores de prevalência com ampla variação - de 1,2 a 13\%. Diferenças metodológicas, número de medidas utilizadas e critérios de referência diferentes são as principais causas dessa variabilidade.
No estudo de Muscatine ${ }^{19}$, a prevalência diminuiu de $13 \%$ para menos de $1 \%$ com o decorrer da pesquisa. Londe, em 1966, nos Estados Unidos, encontrou 12,6\% de crianças hipertensas entre 1.805 avaliadas em consultório. Após o acompanhamento, esse valor caiu para $1,9 \%{ }^{20}$. Adrogué $\&$ Sinaiko, em 2001, avaliando 19.452 crianças de 10 a 15 anos de acordo com os padrões do Task Force de 1996, encontraram 2,7\% com hipertensão sistólica e 2\% com hipertensão diastólica. Reexaminando todas as crianças que tiveram sua pressão arterial acima do percentil 70 , os autores encontraram $0,8 \%$ de hipertensão sistólica e $0,4 \%$ de hipertensão diastólica ${ }^{21}$.

Em relação aos estudos nacionais, também tem sido encontrada grande variabilidade entre as prevalências. Devemos observar, entretanto, que, para caracterizar uma criança como portadora de HAS, são necessárias três medidas de pressão arterial acima do percentil 95 correspondente ao percentil de estatura para idade e sexo deste indivíduo. Essas três medidas deverão ser realizadas em ocasiões diferentes (em dias diferentes) ${ }^{9}$.

Tabela 3 - Prevalência de pressão arterial elevada de estudantes de 7 a 17 anos de Maceió estratificados de acordo com o estado nutricional

\begin{tabular}{lcc}
\hline Estado nutricional (N) & $\begin{array}{c}\text { Prevalência de pressão arterial elevada } \\
\text { Sim }\end{array}$ & Não \\
\hline Normal (1.081) & $8,1 \%(88)$ & $91,9 \%(993)$ \\
Sobrepeso (56) & $28,6 \%(16)$ & $71,4 \%(40)$ \\
Risco de sobrepeso (RSP) (116) & $12,1 \%(14)$ & $87,9 \%(102)$ \\
\hline
\end{tabular}

Pressão arterial elevada normal $\times$ RSP, $\quad$ Fisher bi-caudal, $p=0,1606, \chi^{2}$ não-corrigido, $p=0,2070$

Pressão arterial elevada sobrepeso $\times$ RSP, $\quad$ Fisher bi-caudal, $p=0,0174, \chi^{2}$ não-corrigido, $p=0,0164$

Pressão arterial elevada normal $x$ sobrepeso, Fisher bi-caudal, $p<0,0001, \chi^{2}$ não-corrigido, $p<0,0001$ 
Os resultados do presente estudo demonstram uma prevalência de pressão arterial elevada em 9,4\% dos analisados em uma única oportunidade. Essas crianças deverão ser reavaliadas em mais duas ocasiões para se definir a presença ou não de HAS. Uma vez detectado o problema, o indivíduo deverá ser submetido a uma investigação exaustiva de causas secundárias de hipertensão, principalmente os mais jovens.

\section{Diferenças de pressão arterial entre diferentes faixas etárias}

O aumento da prevalência de níveis tensionais altos nas faixas etárias mais elevadas, encontrado no presente estudo, é esperado, já que a hipertensão essencial (mais comum) tende a ser mais prevalente na pré-adolescência, adolescência e idade adulta19,22,23.

\section{Diferenças de pressão arterial entre estudantes de acordo com o estado nutricional}

O achado de prevalência de pressão arterial elevada maior nos estudantes com sobrepeso é semelhante ao de inúmeros estudos epidemiológicos, nacionais e internacionais, que estudaram a relação entre obesidade e pressão arterial tanto em adultos quanto em crianças e adolescentes, e confirma uma unanimidade científica: a obesidade tem grande impacto sobre a pressão arterial21,24-28.

O presente estudo, o primeiro de cunho epidemiológico realizado em Maceió, aponta para a necessidade de que a medida da pressão arterial seja realmente incorporada à prática clínica pediátrica, de forma que, cada vez mais precocemente, crianças e adolescentes com pressão arterial elevada sejam identificados e avaliados, o que permitirá a detecção e o tratamento de causas secundárias de HAS e de fatores contribuintes para a elevação da pressão arterial, como a obesidade, bem como a intervenção específica nos casos de hipertensão arterial primária. Apenas desta forma poderemos contribuir para a prevenção da epidemia de doenças cardiovasculares prevista pela Organização Mundial de Saúde para os países em transição epidemiológica.

Este trabalho tem como conclusões que a prevalência de pressão arterial elevada foi de $9,4 \%$; não existem diferenças estatisticamente significantes entre as prevalências de pressão arterial elevada entre os gêneros; a diferença entre as prevalências de pressão arterial elevada foi significante quando comparados os grupos escolar e pré-adolescente com o grupo adolescente, e extremamente significante quando comparados os grupos peso normal e sobrepeso.

\section{Referências}

1. Kannel, W. B. Bishop Lecture. Contribution of the Framingham Study to preventive cardiology. J Am Coll Cardiol. 1990;15: 206-11.

2. World Health Organization - Expert Committee on Hypertension Control: Hypertension Control. Report of a WHO Expert Committee, WHO Technical Report Series 862:1-83, Geneva; 1996.
3. Ministério da Saúde do Brasil - Notícias da Saúde, 2000 [site na Internet]. Disponível em: http://portalweb02.saude.gov.br/ saude/aplicacoes/noticias/noticias_detalhe.cfm?co_seq_ noticia $=156$.

4. Task Force on Blood Pressure Control in Children. Report of the Task Force on Blood Pressure Control in Children. Pediatrics. 1977; 59:S797-820.

5. Task Force on Blood Pressure Control in Children. Report of the second Task Force on Blood Pressure Control in Children. Pediatrics. 1987;79:1-25.

6. World Health Organization - Expert Committee on Hypertension Control: Blood Pressure Studies in Children. Report of a WHO Expert Committee, WHO Technical Report Series 715:1-36, Geneva; 1985.

7. World Health Organization - Expert Committee on Hypertension Control: Prevention in childhood and youth of adult cardiovascular diseases: time for action. Report of a WHO Expert Committee, WHO Technical Report Series 792:1-105, Geneva; 1990.

8. Okay Y, Manissadjian A. Comentário - hipertensão arterial. Anais Nestlé. 1986;42:47-54.

9. Task Force on Blood Pressure Control in Children. Update on the 1987 Task Force Report on High Blood Pressure in Children and Adolescents: A Working Group Report from the National High Blood Pressure Education Program National High Blood Pressure Education Program Working Group on Hypertension Control in Children and Adolescents. Pediatrics. 1996;98:649-58.

10. Rosner B, Prineas RJ, Loggie JMH, Daniels SR. Blood pressure nomograms for children and adolescents, by height, sex and age, in the United States. J Pediatr. 1993;123:871-86.

11. Alves JGB, Ribeiro Neto JP, Pacheco THD, Silva LBS. Hipertensão arterial em pré-escolares e escolares da cidade do Recife. J Pediatr (Rio J). 1988;64:336-8.

12. Oliveira RG. Epidemiologia da Hipertensão Arterial em Estudantes de Primeiro e Segundo Graus - O Estudo de Belo Horizonte [dissertação]. Belo Horizonte (MG): Universidade Federal de Minas Gerais; 1995.

13. Sociedade Brasileira de Cardiologia [site na Internet]. SBC Sociedade Brasileira de Cardiologia - 1996 - 2002 [citado 16 de janeiro de 2004]. III Consenso Brasileiro de Hipertensão Arterial, Campos do Jordão, 1998. Disponível em: http:// departamentos.cardiol.br/dha/publicacoes/consenso3/ consen.asp.

14. Hamill PVV, Drizd TA, Johnson CL, Reed RB, Roche AF, Moore WM. Physical growth: National Center for Health Statistic Percentiles. Am J Clin Nutr. 1979;32:607-29.

15. Centers for Disease Control and Prevention [homepage on the Internet]. How to measure weight. Atlanta, CDC [updated April 08, 2003; cited 2004 Jan 16]. Available from: http:// www.cdc.gov/nccdphp/dnpa/bmi/bmi-for-age.htm.

16. Koch VH. Pressão arterial em pediatria: aspectos metodológicos e normatização. Rev Bras Hipertens. 2000;1:71-8.

17. Dean AG, Dean JA, Burton AH, Dicker RC. EpiInfo, versão 1.1.2 - Centers for Disease Control and Prevention: Atlanta, Georgia, \& World Health Organization: Geneva, Switzerland, 2001.

18. National Institutes of Health - Departamento de Saúde e Serviços Humanos dos Estados Unidos. VI Relatório da Reunião do Comitê sobre Prevenção, Deteç̧ão, Avaliação e Tratamento da Hipertensão Arterial, 1997.

19. Clarke WR, Woolson RF, Lauer RM. Changes in ponderosity and blood pressure in childhood: The Muscatine Study. Am J Epidemiol. 1986;124:195-206.

20. Londe S. Blood pressure in children as determined under office condition. Clin Pediatr (Phila). 1966;5:71-8.

21. Adrogué $H E$, Sinaiko AR. Prevalence of hypertension in junior high school-aged children: effect of new recommendations in the 1996 Updated Task Force Report. Am J Hypertens. 2001;14:412-14.

22. McCrory WW. Definition, prevalence, and distribution of causes of hypertension. In: Logie JMH. Pediatric and adolescent hypertension. Boston: Blackwell Scientific Publications; 1992. p. $104-111$.

23. Diniz JSS, Silva JMP, Junior MMM, Heirnger SB, Baitista EN, Figueiredo CL. Hipertensão arterial: estudo retrospectivo em crianças referendadas a uma unidade de nefrologia pediátrica. J Pediatr (Rio J). 1994;70:226-33.

24. Cavalcante JWS. Estudo epidemiológico da pressão arterial de crianças [dissertação] Rio de Janeiro (RJ): Universidade Federal do Rio de Janeiro; 1976. 
25. Lauer RM, Clarke WR, Beaglehole R. Level, trend, and variability of blood pressure during childhood: The Muscatine Study. Circulation. 1984;69:242-9.

26. Sinaiko AR, Donahue RP, Jacobs Jr DR, Prineas RJ. Relation of weight and rate of increase in weight during childhood and adolescence to body size, blood pressure, fasting insulin, and lipids in young adults. The Minneapolis Children's Blood Pressure Study. Circulation. 1999;99:1471-6.

27. Menghetti E, Virdis R, Strambi M, Patriarca V, Riccioni MA, Fossali $\mathrm{E}$, et al. Blood pressure in childhood and adolescence: the Italian normal standards. Study Group on Hypertension of the Italian Society of Pediatrics. J Hypertens. 1999;17:1363-72.
28. He Q, Ding ZY, Fong DY, Karlberg J. Blood pressure is associated with body mass index in both normal and obese children. Hypertension. 2000;36:165-70.

Correspondência:

Adriana Ávila Moura

Rua Eng. Mário de Gusmão, 1149/202

CEP 57035-000 - Maceió, AL

Fone: (82) 327.8521/9973.2243

E-mail: adriana_avila@uol.com.br 